PAPER

\title{
Patterns and severity of neuromuscular transmission failure in seronegative myasthenia gravis
}

\author{
Y Nemoto, S Kuwabara, S Misawa, N Kawaguchi, T Hattori, M Takamori, A Vincent
}

J Neurol Neurosurg Psychiatry 2005;76:714-718. doi: 10.1136/jnnp.2004.043125

See end of article for authors' affiliations

....................

Correspondence to: Satoshi Kuwabara, $M D$, Department of Neurology, Chiba University School of Medicine, 1-8-1 Inohana, Chuo-ku, Chiba, 2608670 Japan; kuwabara-s@faculty. chiba-u.jp

Received 11 April 2004 Revised version received 11 August 2004 Accepted

1 September 2004
Objectives: To compare the clinical and electrophysiological features of myasthenia gravis (MG) patients with (seropositive) or without (seronegative) antibodies to acetylcholine receptor. To investigate whether antibodies to muscle specific kinase (MUSK) and ryanodine receptor (RyR) are associated with particular features.

Methods: Clinical profiles and single fibre electromyography (SFEMG) in the extensor digitorum communis (EDC) were reviewed in consecutive 57 seropositive and 13 seronegative patients. Antibodies to MuSK and RyR were measured by immunoassays.

Results: Of the 13 seronegative patients, four (31\%) were positive for MuSK antibodies and seven (54\%) were positive for RyR antibodies, including all four MuSK positive patients. Clinical features were similar at presentation for seropositive and seronegative patients, but MuSK positive patients frequently developed myasthenic crises. Despite the similar clinical severities at the time of examination, the proportion with positive jitter ( $93 \%$ of seropositive patients, $50 \%$ of MuSK positive patients, and $44 \%$ of MuSK negative patients) and the extent of jitter (mean consecutive difference: $76 \mu \mathrm{s}$ in seropositive patients, $36 \mu \mathrm{s}$ in MuSK positive patients, and $30 \mu \mathrm{s}$ in MuSK negative patients) were less in seronegative MG patients compared with seropositive MG patients.

Conclusions: Seronegative MG is heterogeneous with respect to the presence of antibodies to MuSK. Impairment of neuromuscular synaptic transmission in EDC is less marked in seronegative than seropositive MG despite the similar clinical severity. This discrepancy may partly reflect the distribution of affected muscles in seronegative patients, but it is possible that other factors, such as impaired excitationcontraction coupling resulting from RyR antibodies, contribute to the clinical phenotype.
M yasthenia gravis (MG) is an autoimmune disease of the neuromuscular junction usually mediated by antibodies to acetylcholine receptor (AChR), which are present in $75-94 \%$ of patients with generalised MG (seropositive). ${ }^{1-4}$ AChR antibodies lead to loss of AChRs and single fibre electromyography (SFEMG) shows jitter and blocking of muscle action potentials, indicating a defect in neuromuscular transmission. ${ }^{56}$ Patients without anti-AChR antibody have been referred to as 'seronegative'. Several lines of evidence indicate differences between seronegative and seropositive MG-for example, thymoma is rarely found in seronegative patients. ${ }^{38}$

It is now known that up to $70 \%$ of seronegative $\mathrm{MG}$ patients have antibodies against the muscle specific receptor tyrosine kinase (MUSK). ${ }^{910}$ The extracellular segment of MuSK mediates the agrin-induced clustering of AChRs during synapse formation, and MuSK antibodies reduce the number of AChR clusters in in vitro experiments. ${ }^{11-13}$ MuSK antibodies might, therefore, reduce the number or density of AChRs thereby leading to jitter and blocking of neuromuscular transmission, but detailed SFEMG findings in seronegative patients, with or without MuSK antibodies, have not yet been reported. Moreover, antibodies to other possible muscle antigens, such as titin and ryanodine receptor (RyR), are detected in some MG patients and could have an effect on muscle function, although they have so far only been reported in patients who are seropositive. ${ }^{14-16}$

We studied clinical, electrophysiological, and immunological features of patients with seronegative MG, focusing on patterns and severity of impaired neuromuscular transmission.

\section{METHODS \\ Patients}

Seventy consecutive patients (22 men and 48 women; mean age 49 years) with generalised MG, seen at Chiba University Hospital, Japan, between 2000 and 2003, were studied. For diagnosis of generalised $M G$, we required involvement of both the ocular muscles and bulbar/limb muscles and typical fatigability, with positive response to edrophonium injection, electrophysiological evidence of a defect in neuromuscular transmission (waning on repetitive nerve stimulation test and/or increased jitter on SFEMG), or positive MuSK antibodies. Edrophonium was injected intravenously. Initially $2 \mathrm{mg}$ was given. If this was tolerated and no definite improvement in strength occurred after 30 seconds another $8 \mathrm{mg}$ was injected. A positive test consisted of obvious improvement in blepharoptosis and/or muscle strength, and equivocal improvement was regarded as negative. Patients with purely ocular MG were excluded.

Patients' clinical disabilities were evaluated using the Myasthenia Gravis Foundation of America (MGFA) clinical classification. ${ }^{17}$ At the time of clinical and electrophysiological examination and antibody assays, $73 \%$ of the seropositive and $54 \%$ of the seronegative patients had not yet received thymectomy, corticosteroid treatment, or other immunosuppressive treatments. In patients receiving anticholinesterase medication, this medication was withdrawn 24 hours before the electrophysiological examination.

Abbreviations: $A C h R$, acetylcholine receptor; EDC, extensor digitorum communis; MCD, mean of the absolute consecutive differences; MG, myasthenia gravis; MGFA, Myasthenia Gravis Foundation of America; MuSK, muscle specific kinase; RyR, ryanodine receptor; SFEMG, single fibre electromyography 


\section{Electrophysiology}

Axonal stimulating SFEMG was performed in the extensor digitorum communis (EDC) muscle by the technique described by Trontelij and Stålberg. ${ }^{\circ}$ Stimulation was delivered using a needle cathode (Teflon-coated monopolar steel needle, Nicolet Biomedical Japan, Tokyo, Japan) inserted into the EDC. The stimulation rate was $2 \mathrm{~Hz}$ when searching for optimal action potentials and $10 \mathrm{~Hz}$ during jitter measurements. The SFEMG electrode (Medelec SF 2553031, London, UK) was inserted into the EDC over the area of muscle twitching. Only fibres with an amplitude greater then $0.5 \mathrm{mV}$ and a rise time shorter than $0.25 \mathrm{~ms}$ were accepted. Special care was taken to maintain stimulus intensity above threshold throughout the jitter measurements. Stimulation, recording, and computation of the jitter were performed using a Nicolet Viking 4 EMG machine (Nicolet Biomedical Japan, Tokyo, Japan). Jitter was expressed as the mean of the absolute consecutive differences (MCD) of the latency from the stimulus to the negative peak of the muscle action potential. A series of 100 responses was acquired from each muscle fibre. Between 18 and 24 different muscle action potentials were sampled. The upper normal limits of MCD were $40 \mu$ s for individual motor endplates and $25 \mu$ s for the mean MCD of examined endplates. ${ }^{6}$

Repetitive nerve stimulation test was performed in the nasalis muscle after facial nerve stimulation, in the trapezius after accessory nerve stimulation, and in the abductor digiti minimi after ulnar nerve stimulation. The nerves were stimulated at $3 \mathrm{~Hz}$. The area of the initial negative phase was measured and the percentage decrement was calculated by comparing the fourth response with the first response. An area decrement of more than $10 \%$ was considered abnormal for the abductor digiti minimi, whereas a decrement more than $15 \%$ was seen as abnormal for the nasalis and trapezius muscles because of baseline drift in testing these muscles.

\section{Antibody assays}

AChR antibodies were measured by a standard radioimmunoprecipitation assay using human adult type AChR as antigen. ${ }^{18}$ Serum MuSK antibodies were measured by immunoprecipitation of ${ }^{125}$ I-recombinant MuSK extracellular domains. ${ }^{19}$ Anti-RyR and anti-titin antibodies were determined by enzyme-linked immunosorbent assays. ${ }^{20}$ For anti-RyR assay, peptides corresponding to the C-terminal transmembrane region and $\mathrm{N}$-terminal region were used. Anti-titin assays were performed using recombinant titin fragment (MGT30-peptide, Diagnostika GMBH, Hamburg, Germany).

\section{Statistics}

For statistical analysis, differences in median values were tested by the Mann-Whitney $U$ test, and differences in proportions were tested by the $\chi^{2}$ test or Fisher's exact test.

\section{RESULTS}

\section{Antibodies and clinical profiles}

Anti-MuSK assays were performed in all the 13 seronegative and 57 seropositive patients; four $(31 \%)$ of the seronegative patients and none of the 57 seropositive patients were positive for MuSK antibodies. Anti-RyR and anti-titin assays were performed only in the seronegative patients; seven (54\%) of the 13 seronegative patients were positive for antiRyR antibody, including all four MuSK antibody positive patients. None of the seronegative patients was positive for anti-titin antibody.

Table 1 compares the clinical profiles of 57 seropositive patients, and 13 seronegative patients divided into the MuSK antibody positive $(n=4)$ and negative $(n=9)$ subgroups. On neurological examination, the seronegative patients were indistinguishable from the seropositive patients; seronegative patients also had fluctuating blepharoptosis and/or ophthalmoplegia, and fatigability in the facial, bulbar, or limb muscles. Age, male to female ratio, clinical severity, and distribution of weakness (MGFA Clinical Classification; fig 1) did not differ significantly in the seropositive, MuSK positive, and MuSK negative groups. A positive edrophonium test was less frequent in MuSK negative patients $(\mathrm{p}<0.05)$ and none of the seronegative patients had thymic mass on chest computed tomography compared with $39 \%$ of seropositive patients. On histological findings of the removed thymus, one seronegative/MuSK positive patient had hyperplasia and the thymus was normal in the remaining seronegative patients.

The three subgroups of patients in Table 1 received similar treatments and all experienced clinical improvement after thymectomy, corticosteroid treatment, plasmapheresis, or cholinesterase inhibitors. However, because in most of the patients corticosteroid treatment begun before or within 3 months after thymectomy, the effects of each treatment could not be evaluated. Myasthenic crisis, which was defined as the requirement for intubation because of respiratory weakness, was more frequent in the MuSK posititive patients than in the seropositive MG patients $(\mathrm{p}<0.05$; Table 1$)$.

Table 1 Clinical and laboratory profiles of patients with myasthenia gravis

\begin{tabular}{|c|c|c|c|}
\hline & \multirow{2}{*}{$\frac{\text { Seropositive }}{(n=57)}$} & \multicolumn{2}{|l|}{ Seronegative } \\
\hline & & MuSK positive (n = 4) & MuSK negative $(n=9)$ \\
\hline Age: year, mean (range) & $50(22-84)$ & $40(29-53)$ & $49(20-65)$ \\
\hline Age of onset: year, mean (range) & $47(22-84)$ & $33(22-45)$ & $43(17-64)$ \\
\hline Male:female & 19:38 & $0: 4$ & $3: 6$ \\
\hline MGFA, mean (SD) & $2.4(0.8)$ & $2.8(1.0)$ & $2.0(0.7)$ \\
\hline$a: b$ & $34: 14$ & $2: 2$ & $7: 1$ \\
\hline Positive edrophonium test & $94 \%$ & $75 \%$ & $44 \% *$ \\
\hline Thymic mass on CT & $39 \%$ & $0 \% *$ & $0 \% *$ \\
\hline \multicolumn{4}{|l|}{ Thymus pathology } \\
\hline Normal & $9 / 36(25 \%)$ & $3 / 4(75 \%)$ & $6 / 6(100 \%)$ \\
\hline Hyperplasia & $4 / 36(11 \%)$ & $1 / 4(25 \%)$ & $0 / 6(0 \%)$ \\
\hline Thymoma & $20 / 36(56 \%)$ & $0 / 4(0 \%)$ & $0 / 6(0 \%)$ \\
\hline \multicolumn{4}{|l|}{ Treatment } \\
\hline Thymectomy & $63 \%$ & $100 \%$ & $67 \%$ \\
\hline Corticosteroid & $56 \%$ & $100 \%$ & $45 \%$ \\
\hline Myasthenic crisis & $4 \%$ & $75 \%{ }^{*}$ & $11 \%$ \\
\hline
\end{tabular}



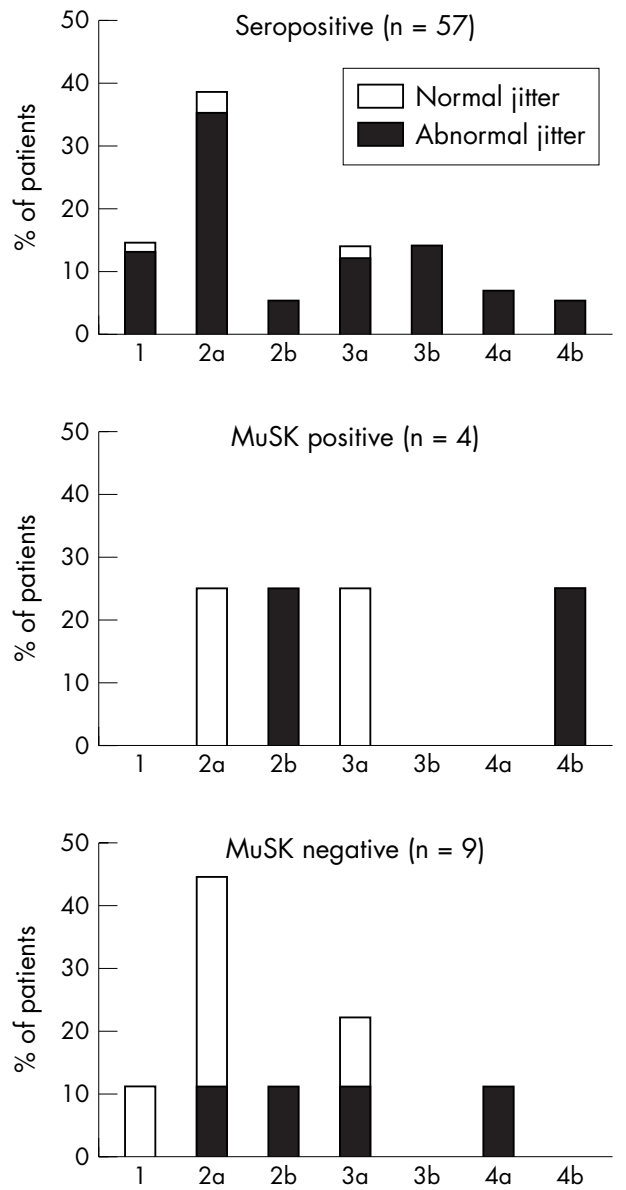

Figure 1 The proportion of patients and the frequency of abnormal jitter according to the clinical classification proposed by Myasthenia Gravis Foundation of America (MGFA). The distribution did not differ significantly among the patient groups, but the percentage of abnormal jitter was lower in the seronegative group with or without muscle specific kinase (MuSK) antibody $(p<0.01)$. Patients classified as having MGFA 1 initially had generalised weakness but showed only ocular symptoms at the time of examination.

\section{Electrophysiology}

Jitter (MCD) was abnormal in EDC in 93\% of seropositive patients but only in $46 \%$ of seronegative patients (Table 2; $\mathrm{p}<0.05$ for both MuSK antibody positive and negative groups), and the number of endplates with increased jitter was also different (Table 2). The proportions of patients with abnormal jitter are shown for each MGFA grade in fig 1 . The most severely affected had abnormal jitter, but a high
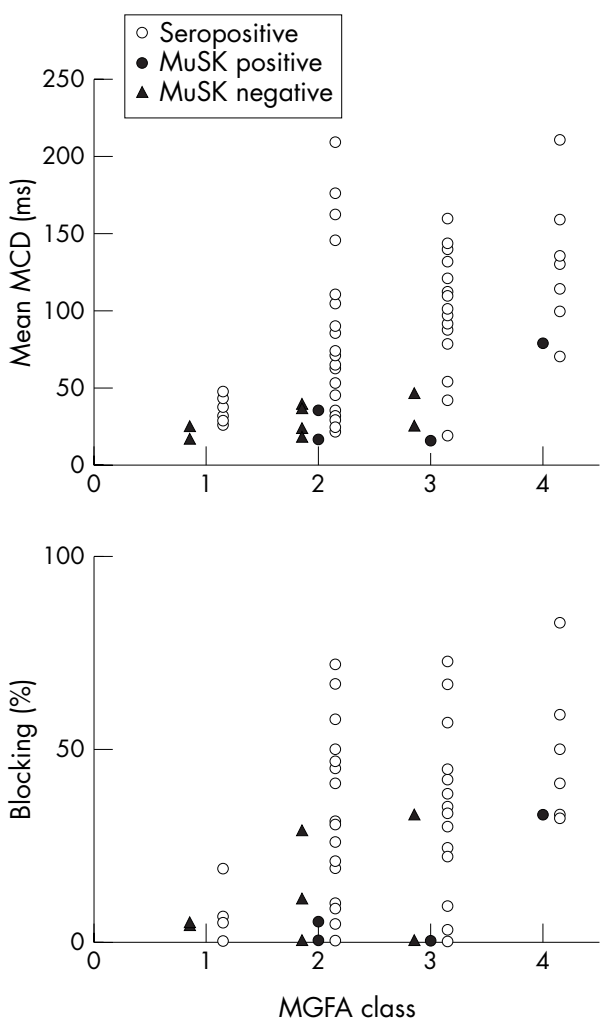

Figure 2 Mean consecutive difference (MCD) and the frequency of endplates with blocking according to the clinical classification proposed by Myasthenia Gravis Foundation of America (MGFA). Patients were divided into three groups according to the antibodies. MuSK, muscle specific kinase.

proportion of seronegative MG patients with MGFA grades of 3a or below exhibited normal jitter. The MCD values and presence of blocking are shown for each group of patients in fig 2. It is clear that, within each MGFA grade, the extent of jitter (mean MCD) and percentage of endplates with blocking were lower for the seronegative group, both with and without MuSK antibodies, compared with the seropositive group.

Because $27 \%$ of seropositive patients and $46 \%$ of the seronegative patients had received treatments at the time of examination, the frequency of abnormal jitter was compared between 'untreated' and 'treated' patients. Among seropositive patients, jitter was abnormal in 95\% of 'untreated' patients and $85 \%$ of 'treated' patients. Among 13 seronegative patients, abnormal jitter was found for two (29\%) of the seven 'untreated' patients and four (67\%) of the six 'treated' patients.

Table 2 Electrophysiology in patients with myasthenia gravis

\begin{tabular}{|c|c|c|c|}
\hline & \multirow{2}{*}{$\begin{array}{l}\text { Seropositive } \\
(n=57)\end{array}$} & \multicolumn{2}{|l|}{ Seronegative } \\
\hline & & MuSK positive $(n=4)$ & MuSK negative $(\mathrm{n}=9$ ) \\
\hline \multicolumn{4}{|l|}{ Single fibre electromyography } \\
\hline Increased jitter & $93 \%$ & $50 \% *$ & $44 \%^{*}$ \\
\hline Mean MCD; mean (SD); $\mu \mathrm{s}$ & $76(51)$ & $36(29)^{*}$ & $30(10)^{*}$ \\
\hline Endplate with $M C D>40 \mu \mathrm{s}$ & $53 \%$ & $20 \% *$ & $18 \%^{*}$ \\
\hline Endplate with blocking & $23 \%$ & $10 \%$ & $10 \% *$ \\
\hline \multicolumn{4}{|c|}{ Repetitive nerve stimulation test (waning) } \\
\hline Nasalis & $23 / 55(41 \%)$ & $2 / 4(50 \%)$ & $2 / 8(25 \%)^{*}$ \\
\hline Trapezius & $19 / 48(40 \%)$ & $1 / 4(25 \%)$ & $1 / 5(20 \%)$ \\
\hline Abductor digiti minimi & $15 / 46(33 \%)$ & $1 / 4(25 \%)$ & $1 / 5(20 \%)$ \\
\hline Any in the three muscles & $67 \%$ & $50 \%$ & $25 \% *$ \\
\hline
\end{tabular}


Table 3 Laboratory findings of patients with seronegative myasthenia gravis

\begin{tabular}{|c|c|c|c|c|c|c|c|c|}
\hline \multirow[b]{2}{*}{ Patient } & \multirow[b]{2}{*}{ Age, sex } & \multirow[b]{2}{*}{ MGFA class } & \multicolumn{2}{|c|}{ Antibodies to: } & \multirow[b]{2}{*}{ Edrophonium test } & \multirow{2}{*}{$\begin{array}{l}\text { Repetitive stimulation } \\
\text { test }\end{array}$} & \multicolumn{2}{|c|}{ Single fibre electromyography } \\
\hline & & & MuSK & RyR & & & Jitter* (mean MCD) & Blockingt (\%) \\
\hline 1 & $53, \mathrm{~F}$ & $2 a$ & + & + & + & - & 16 & 0 \\
\hline 2 & $39, \mathrm{~F}$ & $3 a$ & + & + & - & - & 15 & 0 \\
\hline 3 & $39, \mathrm{~F}$ & $2 b$ & + & + & - & + & 35 & 5 \\
\hline 4 & $29, \mathrm{~F}$ & $4 \mathrm{~b}$ & + & + & + & + & 78 & 33 \\
\hline 5 & $61, M$ & $2 a$ & - & + & - & - & 25 & 0 \\
\hline 6 & $51, \mathrm{~F}$ & $2 b$ & - & + & - & + & 36 & 29 \\
\hline 7 & $36, M$ & $2 a$ & - & + & + & - & 23 & 0 \\
\hline 8 & $30, \mathrm{~F}$ & 1 & - & - & + & - & 17 & 0 \\
\hline 9 & $20, F$ & $2 a$ & - & - & + & - & 18 & 0 \\
\hline 10 & $65, \mathrm{~F}$ & $2 a$ & - & - & - & - & 39 & 0 \\
\hline 11 & $61, \mathrm{~F}$ & $3 a$ & - & - & - & + & 25 & 0 \\
\hline 12 & $65, M$ & $3 a$ & - & - & - & - & 37 & 11 \\
\hline 13 & $49, \mathrm{~F}$ & $4 a$ & - & - & + & + & 46 & 33 \\
\hline
\end{tabular}

MCD, mean consecutive difference; MGFA, clinical score by Myasthenia Gravis Foundation of America; MuSK, muscle specific kinase; RyR, ryanodine receptor; +, positive; -, negative. " normal, $25 \mathrm{~ms}$ or less; tnormal, $0 \%$. Electrophysiological studies were performed before treatment in patients $2,5,7,8,10,11,13$.

Table 2 also shows results of the repetitive nerve stimulation test, which was performed in three muscles. In the seropositive patients, waning was present in at least one examined muscle in $67 \%$ of patients. Two of the four MuSK positive patients showed waning in the nasalis, whereas waning in the MuSK negative group was found in only $25 \%$. Overall, there was significantly less waning in the MuSK negative patients compared with the seropositive $M G$ patients $(\mathrm{p}<0.05)$.

Table 3 details the findings in the 13 patients with seronegative MG with the patients listed according to the positivities of MuSK and RyR antibodies. Electrophysiological examination was performed before treatment in seven patients (patient 2, 5, 7, 8, 10, 11, 13) and after treatment in the remaining six patients. Ten of the 13 patients had clinical involvement of the EDC; nine patients graded as having MGFA ' $a$ ' had weakness in the forearm muscles, and patient 4 was graded as having MGFA ' $4 b^{\prime}$ ' but had involvement of EDC. Of the four MuSK positive patients (patients 1-4), two showed mild-to-moderate jitter and the remaining two had normal SFEMG findings. Of the seven anti-RyR positive patients (patients 1-7), three showed an increased jitter and the remaining four had normal jitter. Similarly, mild or no SFEMG abnormalities were present for the other seronegative MG patients (patients 8-13). Therefore, MuSK antibodies or anti-RyR antibodies were not associated with specific patterns of SFEMG abnormalities.

\section{DISCUSSION}

Our results showed a number of differences in clinical, electrophysiological, and immunological profiles between seronegative and seropositive MG. First, consistent with previous study results, ${ }^{38}$ thymoma was less common in the seronegative group. Second, seronegative patients had less severe SFEMG abnormalities in the forearm muscles than seropositive patients despite the similar clinical severities and distribution of weakness. This suggests that seronegative MG patients had milder impairment of neuromuscular synaptic transmission in some muscles. Third, in this Japanese series, MuSK antibody was present in only $31 \%$ of the seronegative MG patients whereas anti-RyR antibody was found in 54\% of the patients. The MuSK positive patients frequently have myasthenic crises, but SFEMG abnormalities were mild at the times tested, and in the muscles examined, and there was no clear influence of either MuSK antibodies or RyR antibodies with respect to the electrophysiological investigations in this small series of seronegative MG.
Measuring jitter with SFEMG is the most sensitive in vivo test of abnormal neuromuscular transmission and, therefore, the most sensitive test for MG. ${ }^{6}{ }^{21}$

In the EDC muscle, an increased jitter is found for approximately $90 \%$ of patients with generalised MG and for $78 \%$ of patients in clinical remission after treatment. ${ }^{21}$ In this series, abnormal jitter in the EDC was observed in 93\% of the seropositive patients but only in $46 \%$ of the seronegative patients. We do not have control data for our jitter studies but the values that we obtained for seropositive patients are similar to those reported by others, and the lack of control data does not affect the difference between seropositive and seronegative patients that we found.

The extent of increased jitter was less in the patients with seronegative MG. A similar finding (less jitter for seronegative $M G$ ) was reported in a previous study but detailed data were not shown. ${ }^{3}$ Other recent reports investigating MuSK antibody positive patients suggest that jitter was normal in the EDC, but increased in shoulder and neck muscles or facial muscles. ${ }^{12}{ }^{13}$ Our SFEMG study included only the EDC muscle and the possibility of abnormal jitter in other muscle-for example, facial muscles-could not be excluded. Moreover, it is theoretically possible that prior treatment reduced the frequency of abnormal jitters in the seronegative patients but our data do not support this possibility.

These findings raise the interesting possibility that the neuromuscular junction is not the only target of the immune attack in seronegative MG, and that the similar clinical severity for seropositive and seronegative patients could be because other factors contribute to myasthenic symptoms in seronegative patients. A myasthenic-like syndrome without abnormality in neuromuscular synaptic transmission has been demonstrated in the Buffalo/Mna rat, which have elevated serum anti-RyR antibodies. ${ }^{22}{ }^{23}$ The rats begin to develop muscle weakness soon after birth and a physiological study showed reduction of muscle contractile force without abnormalities in synaptic transmission or membrane properties, perhaps caused by defective release of $\mathrm{Ca}^{2+}$ from the sarcoplasmic reticulum. ${ }^{23}$ In theory, a similar disturbance of E-C coupling might be responsible for some of the muscle weakness in seronegative MG patients who also have RyR antibodies; however, there was no evidence that the patients with RyR antibodies had greater electrophysiological abnormalities than those without. Future studies will need to investigate neuromuscular transmission in different muscle groups and to identify the pathophysiology of each subtype of seronegative MG. 


\section{ACKNOWLEDGEMENT}

This study was supported in part by a research grant for Neuroimmunological Diseases from the Ministry of Health, Labor and Welfare of Japan (SK), and from the Myasthenia Gravis Association and Muscular Dystrophy Campaign of Great Britain (AV).

\section{Authors' affiliations}

Y Nemoto, S Kuwabara, S Misawa, N Kawaguchi, T Hattori, Department of Neurology, Chiba University School of Medicine, Chiba, Japan

M Takamori, Neurological Center, Kanazawa-Nishi Hospital, Kanazawa, Japan

A Vincent, Department of Clinical Neurology, Weatherall Institute of Molecular Medicine, University of Oxford, London, UK

Competing interests: none declared

\section{REFERENCES}

1 Lindstorm JM, Seybold ME, Lennon VA, et al. Antibody to acetylcholine receptor in myasthenia gravis. Neurology 1976;26:1054-9.

2 Lefvert AK, Bergstorm K, Matell G, et al. Determination of acetylcholine receptor antibody in myasthenia gravis: clinical usefulness and pathogenetic implications. J Neurol Neurosurg Psychiatry 1978;41:394-403.

3 Soliven BC, Lange DJ, Penn AS, et al. Seronegative myasthenia gravis. Neurology 1988:38:514-6.

4 Sanders DB, Andrewa PI, Howard JF Jr, et al. Seronegative myasthenia gravis. Neurology 1997;48(Suppl 5):S40-5.

5 Stålberg E. Clinical electrophysiology in myathenia gravis. J Neurol Neurosurg Psychiatry 1980;43:622-33.

6 Trontelj JV, Stålberg E. Jitter measurement by axonal micro-stimulation. Guidelines and technical notes. Electroencephal Clin Neurophysiol 1992:85:30-7.

7 Verma OK, Oger JJ. Seronegative generalized myasthenia gravis: low frequency of thymic pathology. Neurology 1992;42:586-9.

8 Vincent A, Li Z, Hart A, et al. Seronegative myasthenia gravis. Evidence for plasma factor(s) interfering with acetylcholine receptor function. Ann NY Acad Sci 1993:681:529-38.
9 Hoch W, Mcconville J, Helms S, et al. Auto-antibodies to the receptor tyrosine kinase MuSK in patients with myasthenia gravis without acetylcholine receptor antibodies. Nat Med 2001;7:365-8.

10 Liyanage $Y$, Hoch W, Beeson D, et al. The agrin/muscle specific kinase pathway: New target for autoimmune and genetic disorders at the neuromuscular junction. Muscle Nerve 2002;25:4-16.

11 Vincent A, Bowen J, Newsom-Davis J, et al. Seronegative generalized myasthenia gravis: clinical features, antibodies and their targets. Lance Neurology 2003;2:99-106.

12 Evoli A Tonali PA, Padua L, et al. Clinical correlates with anti-MuSK antibodies in generalized seronegative myasthenia gravis. Brain 2003; 126:2304-11.

13 Sanders DB, El-Salem K, Massey JM, et al. Clinical aspects of MuSK antibody positive seronegative MG. Neurology 2003;60:1978-80.

14 Buckley C, Newsom-Davis J, Willcox N, et al. Do titin and cytokine antibodies in $M G$ patients predict thymoma or thymoma recurrence? Neurology $2001 ; 57: 1579-82$

15 Mygland A, Aarli JA, Matre R, et al. Ryanodine receptor antibodies related to severity of thymoma associated myasthenia gravis. I Neurol Neurosurg Psychiatry 1994;57:843-6.

16 Skeie GO, Mygland A, Aarli JA, et al. Titin antibodies in patients with late onset myasthenia gravis: clinical correlations. Autoimmunity 1995;29:99-104

17 Jaretzki A, Barohn RJ, Ernstoff RM, et al. Task Force of the Medical Scientific Advisory Board of the Myasthenia Gravis Foundation of America, Myasthenia gravis: recommendations for clinical research standard. Neurology 2000;55:16-23.

18 Beeson D, Jacobson L, Newsom-Davis J, et al. A transfected human muscle cell line expressing the adult subtype of the human muscle acetylcholine receptor for diagnostic assays in myasthenia gravis. Neurology 1996;47:1552-5

19 McCorville J, Farugia ME, Beeson D, et al. Detection and characterization of MuSK antibodies in seronegative myasthenia gravis. Ann Neurol 2000;55:580-4.

20 Takamori M. Immunology of ryanodine receptor and FK506 in myasthenia gravis. Curr Top Peptide Protein Res 2003;5:187-99.

21 Sanders DB. Clinical impact of single-fiber electromyography. Muscle Nerve 2002;(Suppl 11):S15-20.

22 Iwasa K. Striational autoantibodies in myasthenia gravis mainly react with ryanodine receptor. Muscle Nerve 1997;20:753-6.

23 Iwasa K, Komai K, Takamori M. Spontaneous thymoma rat as a model for myesthenic weakness caused by anti-ryanodine receptor antibodies. Muscle Nerve 1998;21:1655-60. 